\title{
Microsolvation effects on the excited state dynamics of protonated tryptophan
}

Sébastien R. Mercier, ${ }^{\dagger}$ Oleg V. Boyarkin,$^{\dagger} *$ Anthi Kamariotis, ${ }^{\dagger}$ Matteo Guglielmi, ${ }^{\ddagger}$ Ivano

Tavernelli, ${ }^{\ddagger}$ Michele Cascella, ${ }^{\dagger}$ Ursula Rothlisberger, ${ }^{\dagger}$ and Thomas R. Rizzo ${ }^{\dagger} *$

Institute of Chemical Sciences and Engineering, École Polytechnique Fédérale de

Lausanne, CH-1015 Lausanne, Switzerland

\section{Supporting information:}

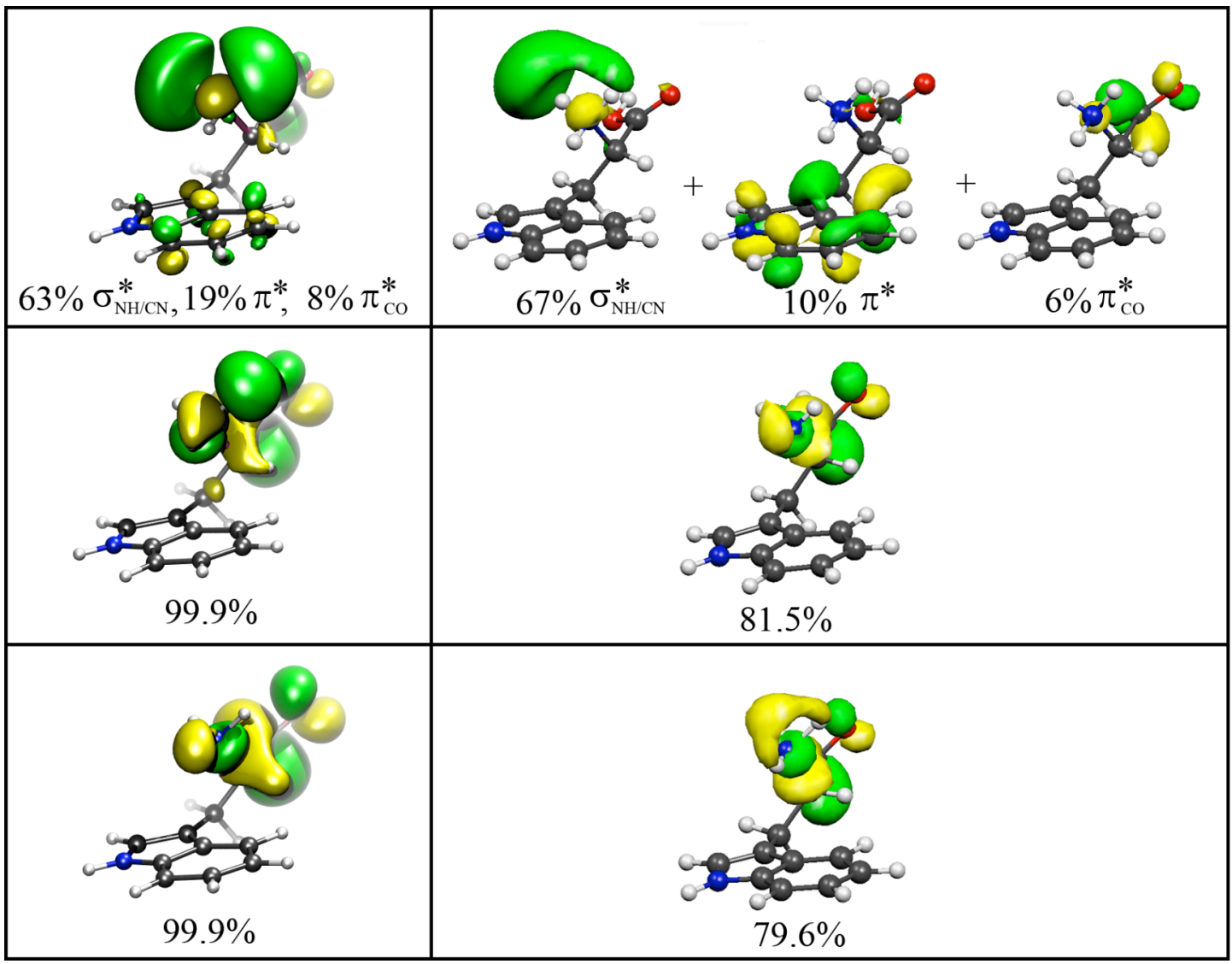

Figure S1. Orbital character for the populated excited state along the molecular dynamics run taken at the first three time-frames labeled with circles in figure $3 \mathrm{~b}$. Left column: TDDFT orbitals, right column: corresponding RI-CC2/aug-ccpVDZ orbitals. 